\title{
ESTADO DE CONOCIMIENTO DE LAS ALGAS DULCEACUICOLAS DE CHILE (EXCEPTO BACILLARIOPHYCEAE)
}

\author{
CURRENT STATE OF KNOWLEDGE OF FRESHWATER ALGAE OF CHILE \\ (EXCEPT BACILLARIOPHYCEAE)
}

\author{
Oscar Parra \\ Unidad de Sistemas Acuáticos, Centro de Ciencias Ambientales, EULA-Chile; Departamento de Botánica, \\ Facultad de Ciencias Naturales y Oceanográficas, Universidad de Concepción, Chile. Email: oparra@udec.cl
}

\begin{abstract}
RESUMEN
Se hace una revisión de la información taxonómica de las algas dulceacuícolas de Chile, particularmente referida a la diversidad expresada como riqueza específica de los grupos de algas que han sido citados para el país, exceptuando al grupo de las diatomeas o Bacillariophyceae. El análisis comprende desde las primeras citas bibliográficas a un análisis de la información existente. Se entrega un inventario de los géneros y especies citados por clase de algas, los tipos de comunidades, los cuerpos de aguas más estudiados, y se describen también algunas contribuciones que han significado un aporte al conocimiento taxonómico y florístico de estos grupos de algas. Gran parte de la información taxonómica ha derivado de estudios que en la mayoría de los casos tuvieron objetivos más ecológicos o limnológicos. Como consecuencia de lo anterior, lo que se conoce de la flora algal de los ambientes acuáticos continentales de Chile representa una subestimación de la riqueza específica. Actualmente, no existen en el país especialistas o grupos de especialistas que prioricen los objetivos taxonómicos en la investigación de ambientes acuáticos continentales, por las dificultades que se tienen para la obtención de recursos que financien estas investigaciones, o la escasa formación de especialistas a nivel de postgrados.
\end{abstract}

Palabras claves: Microalgas, sistemas dulceacuícolas, biodiversidad, Chile.

\begin{abstract}
This paper is a review of taxonomical information of freshwater algae of Chile, particularly referred to diversity expressed as specific richness of the algal groups cited for the country, except for the diatoms group or Bacillariophyceae. The analysis comprises the first bibliographical citations and an analysis of current information on algal biodiversity. An inventory has been given for the genera and species cited per algal class, the types of communities, the most studied water bodies, and are also described some contributions which have provided a support for the taxonomic and floristic knowledge of these algal groups. Great part of taxonomical information have derived from the studies which in the majority of the cases had most ecological and limnological objectives. Consequently, what it is known on the algal flora of continental aquatic environments of Chile represents an underestimation for the specific richness. At present, it does not exist in the country specialists or specialist groups preceding in time the taxonomic objectives in the continental aquatic environments, for the difficulties found to obtain funding resources for investigations or specialists formation at a postgraduate level. In the same way with the great majority of organism groups.
\end{abstract}

KeYwords: Microalgae, freshwater ecosystems, biodiversity, Chile. 
Algas dulceacuícolas de Chile: PARrA, O.

\section{INTRODUCCION}

El término Algae, para designar una entidad taxonómica, fue usado por primera vez por Linnaeus en 1753, en su trabajo "Species plantarum", cuando así designó a uno de los cuatro órdenes propuestos para las criptógamas. La mayor parte de lo que Linnaeus denominó como Algae incluyó, en verdad, a hepáticas y líquenes (Parra \& Bicudo 1996). El término Algae, tal como es comprendido hoy, está desprovisto de significado taxonómico y no corresponde, por tanto, a ninguna categoría nomenclatural. Es simplemente un término colectivo para un grupo de plantas extremadamente heterogéneo, tornando difícil y también problemática su definición.

El número exacto de especies de organismos conocidos para la ciencia permanece desconocido; las cifras que se indican tienen grandes diferencias. El número de especies descritas, según la más reciente publicación sobre biodiversidad, varía entre 1,4 millones a 1,8 millones (Hammond 1995). Para las algas, Wilson (1992) estimó en 26.900, el número de especies actuales descritas, mientras que Hammond (1995) describe un número superior a 40.000 especies. Este último número, sin embargo, se entiende como la estimación más reciente actualizada, ya que corresponde a la opinión de varios especialistas consultados, a revisiones críticas de publicaciones y a ajustes con nueva información (Bicudo \& Bicudo 1996). El mismo Hammond (1992) estima que las algas corresponden al 2,4\% de todas las especies de organismos actualmente conocidas. En todo caso, en lo que concierne a las algas dulceacuícolas, Bourrelly (1972) menciona 13.500 especies descritas.

Parra y Bicudo (1996) reconocen 17 clases de algas, las cuales son diferenciadas por seis criterios fundamentales, a saber: pigmentos fotosintetizadores, estructura del cloroplasto, naturaleza química de la pared celular, naturaleza química y almacenamiento de los productos de reserva, características asociadas al aparato flagelar y características citológicas.

A pesar que el Código de Nomenclatura Botánica reconoce a la división como la categoría taxonómica superior para la clasificación de los organismos fotosintetizadores o derivados de ellos, las divisiones de algas han sufrido en las últimas décadas grandes variaciones o cambios, producto de lo cual han surgido una cantidad de sistemas de clasificación de algas a nivel de divisiones (Parra \& Bicudo 1996). El nivel de clasificación de clases ha resultado en el tiempo más permanente y de más fácil referencia a la hora de analizar el conocimiento taxonómico de este grupo de organismos.

Así como en este trabajo es necesario adoptar algún sistema de clasificación, es también útil tener en consideración los diversos tipos de comunidades algales que han sido descritos (Round 1973, Parra \& Bicudo 1996). Las dos comunidades genéricas, básicas, corresponden a las algas dulceacuícolas planctónicas y bentónicas; en ambas es factible reconocer subcomunidades. El fitoplancton corresponde a la comunidad de organismos microscópicos fotosintetizadores que se hallan en suspención en las aguas y que pueden ser delimitadas ecológica y fisionómicamente. Por otra parte, el fitobentos corresponde al complejo de comunidades algológicas fotoautotróficas que viven asociadas a un sustrato del fondo de los cuerpos de aguas. En el fitobentos es factible distinguir las siguientes subcomunidades de algas:

(a) algas epipélicas, que son aquellas que crecen sobre el sedimento; (b) algas epilíticas, las que crecen sobre superficies rocosas; (c) algas epifiticas, que son las que crecen sobre superficies de vegetales, (d) algas epizoicas, las que crecen sobre superficies animales; (e) algas endófitas, las que crecen dentro de las células o tejidos vegetales; y (f) algas episámmicas, que son las algas que crecen sobre los granos de arenas.

También se reconoce como una comunidad independiente la que se denomina metafiton, la cual está integrada por algas que se encuentran agregadas en la zona litoral, la cual no está estrictamente adherida a un sustrato ni es verdaderamente planctónica. Por otra parte el término perifiton, generalmente se refiere a una microflora algal que crece sobre un substrato, ya sea natural o artificial. El presente trabajo se focaliza en la riqueza de especies, teniendo en cuenta que este parámetro comunitario es una de las medidas más frecuente de biodiversidad usada. Es importante tener en cuenta que el concepto de "diversidad de especies" comúnmente usado en ecología, el cual toma en cuenta tanto el número de especies (riqueza específica), como su importancia relativa en la comunidad (equidad), no es el objetivo de esta publicación. 
BREVE RESEÑA HISTÓRICA DE LOS ESTUDIOS DE ALGAS

Resulta interesante constatar que las primeras citas corresponden a material algológico recolectado en el extremo sur del país, i.e, Tierra del Fuego, Patagonia y la Antártica, dándose un patrón muy similar al acontecido para la flora y la fauna terrestre. Entre estos trabajos se puede mencionar los de Wille (1884), De Toni (1889), Borge (1901), Daday (1902) y Gain (1911a,b). Posteriormente, destacan los trabajos de Izquierdo (1906), Espinoza $(1917,1923)$ y Perez Canto (1929), que se refieren a colecciones algológicas realizadas en la región de Santiago, de charcos y suelos, más los trabajos pioneros de Schwabe (1936) en algas de suelos y biotopos termales.

Sin embargo, los estudios florísticos algales dulceacuícolas se inician con los trabajos de Thomasson (1955 y 1963), en ecosistemas acuáticos de Tierra del Fuego y en los lagos Nordpatagónicos, respectivamente. Estos últimos fueron y bautizados como "Lagos Araucanos" por el mismo Thomasson (1963). En la década del 60 están los trabajos de Asprey et al. (1964) sobre el fitoplancton de aguas dulce de Sudamérica, y el de Forest \& Weston (1966) con las primeras citas de algas del desierto de Atacama. Posteriormente, Navarro \& Avaria (1971) publican uno de los primeros trabajos para lagos en la parte central del país, específicamente sobre el fitoplancton del lago Peñuelas.

A partir del año 1973, Parra y colaboradores inician el aporte más sistemático al conocimiento de la diversidad algológica de agua dulce del país, centrándose primeramente en estudios estrictamente florísticos de los sistemas lacustres y fluviales de la Región del Bío-Bío (Parra 1973, 1975, 1977a,b, Parra et al. 1973, 1976, Rivera et al. 1973), para posteriormente ampliar esta cobertura geográfica a cuerpos de aguas de la región centro sur del país, del Bío-Bío al río Cachapoal (Pereira \& Parra 1984), de Chiloé (Parra \& González 1978), Juan Fernández (Parra \& González 1982) y del sistema de lagos de Torres del Paine (Theoduloz \& Parra 1984). Este mismo grupo de investigación, a partir del año 1976, da inicio a los estudios limnológicos de los lagos de la Región del Bío-Bío, estudios que incluye el componente fitoplanctónico; así se publican los estudios de Parra (1977), Parra et al. (1976) y Dellarrosa et al. (1976), sobre las lagunas "Chica de San Pedro", "La Posada” y "Lo Méndez”, respectivamente; Parra et al. (1989) y Parra (1989), sobre las lagunas intraurbanas de Concepción y la laguna Grande de San Pedro; Parra et al. (1999), Parra y Bicudo (1986) para el sistema de "lagos Nahuelbutanos" (Parra et al. 2003). Dos publica-ciones en este período, (Parra et al. 1986 y Parra y Dellarossa 1987), dan cuenta de floraciones algales tóxicas y no tóxicas en las lagunas de Concepción. Parra et al. (1993), en el estudio limnológico de los lagos Icalma y Galletué, incluyen la descripción de las respectivas comunidades fitoplanctónicas de esos cuerpos de aguas.

La mayoría de los estudios anteriores se referían a las comunidades fitoplanctónicas. A partir de los años 80, el grupo inicia su trabajo en comunidades bénticas de algunos lagos y ríos; entre ellos se destacan los de Pereira y Parra (1984) y Parra et al. (1984), sobre fitobentos de lagos y ríos localizados entre Concepción y Santiago, y el de Basualto et al. (1992), sobre el fitoplancton del río Bío-Bío.

A partir del año 1982, nuestro grupo inicia su colaboración con el grupo de investigación del Dr. Hugo Campos (q.e.p.d.). Producto de esta alianza se desarrollaron entre los años 1987-1995 estudios limnológicos, incluyendo estudios del fitoplancton de los lagos araucanos. Estos generaron las siguientes publicaciones: Campos et al. (1987a), lago Caburgua; Campos et al.(1987b), lago Riñihue; Campos et al. (1988), lago Llanquihue; Campos et al. (1989), lago Puyehue; Campos et al.(1990), lago Todos los Santos; Campos et al. (1992a), lago Rupanco, y Campos et al. (1992b), lago Ranco.

En el mismo marco de colaboración científica se inicia, a partir del año 1994, las publicaciones generadas por el proyecto de investigación científica dirigido por Doris Soto, que permitió el estudio del firoplancton del sistema de lagos del Parque Nacional "Torres del Paine", con publicaciones de Soto et al. (1994); Campos et al. (1994a), lago Toro; Campos et al. (1994b), lago Sarmiento; Campos et al. (1996), laguna Amarga y Villalobos et al. 2003 a y b; Woelfl et al. 2003 a y b; para los lagos Chapo, lagos de Chiloé y Riñihue.

Otros aportes relevante para el conocimiento algal de ambientes dulceacuícolas corresponden a los de Vila y colaboradores (Vila et al. 1987, 1997, 2000; Vila \& Pardo 2003, Mülhauser et al. 1995) para los lagos Rapel y Chungará y los de Parra et. al. (1990) y Cifuentes et al. (1992), para los ambientes hipersalinos de la Región de Atacama en el norte de Chile. 
Por último, se debe destacar el esfuerzo realizado por el grupo de investigación de la Universidad de Concepción orientado a compilar la información taxonómica existente, para que sea utilizada como punto de partida y guía por los interesados en estos grupos de organismos. Así se publican por Parra y González $(1976,1977)$, dos catálogos que incluyen información sobre las Cyanophyceae, el primero y Pyrrophyta, Chrysophyceae, Xanthophyceae, Rhodophyta, Euglenophyta y Chlorophyta, el segundo. Posteriormente, entre los años 1982 y 1983 se publica por Parra O., M. González, V. Dellarossa, P. Rivera \& M. Orellana, el "Manual Taxonómico del Fitoplancton de Aguas Continentales, con especial referencia al fitoplancton de Chile". Es una obra de cinco volúmenes, editado por la Universidad de Concepción y que incluye en el Vol. 1: Cyanophyceae, Vol. 2: Chrysophyceae-Xantho-phyceae, Vol. 3: Cryptophyceae, Dinophyceae y Euglenophyceae, Vol. 4: Bacillariophyceae y el Vol. 5: (partes 1 y 2), Chlorophyceae. Otro esfuerzo realizado en esta misma dirección fue la obra de Parra y Bicudo (1996), titulada "Algas de Aguas Continentales: Introducción a la Biología y Sistemática", también editada por la Universidad de Concepción y que se realizó pensando en una obra introductoria para aquellos que se querían iniciar en el estudio taxonómico de este grupo de organismos. Sin embargo, hay que reconocer que un catálogo de toda las especies de algas dulceacuícolas al día de hoy no existe para nuestro país.

\section{RESULTADOS}

INVENTARIO TAXONÓMICO DE LAS ALGAS DE AGUA DULCE DE CHILE

La Tabla I sintetiza la información taxonómica existente en el país. De ella se puede inferir que de las 17 clases reconocidas (16 sin incluir a las Bacillariophyceae), 12 de ellas están representadas en Chile, con un total de 190 géneros y 1325 especies. Las clases de algas con mayor riqueza específica conocida, corresponden a las Chlorophycaeae, Chrysophyceae, Cyanophyceae, Xanthophyceae y Dinophycaeae. Los géneros con mayor número de especies se presentan en la Tabla II.
TABLA I. Número de géneros y especies de algas de aguas continentales citados para Chile.

TABLE I. Number of genus and species of freshwater algae cited for Chile.

\begin{tabular}{lcc}
\hline Clases & Géneros & Especies \\
\hline Cyanophyceae & 18 & 23 \\
Chrysophyceae & 14 & 72 \\
Xanthophyceae & 23 & 63 \\
Cryptophyceae & 6 & 20 \\
Rhodophyceae & 2 & 2 \\
Dinophyceae & 7 & 31 \\
Euglenophyceae & 16 & 103 \\
Chlorophyceae & 101 & 905 \\
Charophyceae & 2 & 6 \\
Total & 189 & 1.325 \\
\hline
\end{tabular}

\section{CONCLUSIONES}

Se concluye que la información taxonómica disponible a nivel de riqueza específica para el país se caracteriza por lo siguiente: (1) Las recolecciones o muestreos de material algológico han sido invariablemente puntuales, esto es, muchas de las floras algales de cuerpos de aguas dulceacuícolas fueron el resultado de recolecciones en un sitio de muestreo, por lo tanto no representativas de las variaciones espaciales y temporales; (2) Las recolecciones o muestreos, en su mayoría, corresponden a material planctónico, recolectado de diversas maneras; (3) Una parte importante de las muestras han sido tomada de las orillas de los cuerpos de aguas, donde se puede encontrar representado una mezcla de comunidades, que van desde el plancton de orillas (tichoplankton) hasta algas que crecen en comunidades asociadas a algas filamentosas (metaphyton); (4) Una parte muy importante del material algológico citado ha correspondido a material recolectado en estudios ecológicos, que ha comprendido a muestreos, de frecuencia mensual o estacional en un ciclo anual, y con muestras tomadas generalmente en una estación y a diversas profundidades de cuerpos de aguas lacustres; (5) Por otra parte, las recolecciones de comunidades de algas bénticas, corresponden a estudios más recientes, los que incluyen sustratos naturales y sustratos artificiales; (6) La mayoría de los trabajos limnológicos entregan sólo los listados de las especies, raras veces ilustraciones, así como 
tampoco indicaciones de las fuentes bibliográficas que sirvieron de base de la identificación taxonómica y sin preservar el material algológico.

En resumen, gran parte de la información taxonómica que se tiene de los diversos grupos de algas en Chile se ha derivado de estudios que en la mayoría de los casos tuvieron objetivos más ecológicos o limnológicos, los cuales se orientaban más a caracterizaciones generales de lagos o ríos o a responder hipótesis científicas sobre determinados procesos o compartimentos de los ecosistemas estudiados. Como consecuencia de lo anterior, lo que se conoce de la flora algal de los ambientes acuáticos continentales de Chile representa una subestimación de la riqueza específica.

Actualmente no existen en el país especialistas o grupos de especialistas que prioricen los objetivos taxonómicos en la investigación de ambientes acuáticos continentales, por las dificultades que se tienen para la obtención de recursos que financien estas investigaciones. Al igual que para la gran mayoría de los grupos de organismos, se hace necesario la formulación de un plan específico orientado a estimular y proveer con recursos específicos la investigación y la formación de especialistas en taxonomía de estos grupos.

TABLA II. Listado de géneros con mayor número de especies en Chile.

TABLE II. Genera list with the major number of species in Chile.

\begin{tabular}{|c|c|c|}
\hline Clases & Géneros & Especies \\
\hline \multirow{5}{*}{ Cyanophyceae } & Anabaena & 16 \\
\hline & Lyngbya & 10 \\
\hline & Microcystis & 10 \\
\hline & Oscillatoria & 24 \\
\hline & Spirulina & 10 \\
\hline \multirow[t]{4}{*}{ Chrysophyceae } & Chromulina & 6 \\
\hline & Dinobryon & 9 \\
\hline & Mallomonas & 20 \\
\hline & Synura & 8 \\
\hline \multirow[t]{4}{*}{ Xanthophyceae } & Tribonema & 11 \\
\hline & Ophiocytium & 5 \\
\hline & Pseudostauratrum & 4 \\
\hline & Tetraplektron & 4 \\
\hline \multirow[t]{3}{*}{ Cryptophyceae } & Rhodomonas & 5 \\
\hline & Cryptomonas & 7 \\
\hline & Chroomonas & 3 \\
\hline Rhodophyceae & Compsopogon & 2 \\
\hline \multirow[t]{3}{*}{ Dinophyceae } & Gymnodinium & 9 \\
\hline & Peridinium & 13 \\
\hline & Ceratium & 2 \\
\hline \multirow[t]{3}{*}{ Euglenophyceae } & Euglena & 16 \\
\hline & Phacus & 17 \\
\hline & Trachellomonas & 25 \\
\hline \multirow[t]{14}{*}{ Chlorophyceae } & Ankistrodesmus & 8 \\
\hline & Chlamydomonas & 8 \\
\hline & Dunaliella & 3 \\
\hline & Coelastrum & 4 \\
\hline & Oocystis & 10 \\
\hline & Pediastrum & 11 \\
\hline & Scenedesmus & 43 \\
\hline & Tetraedron & 9 \\
\hline & Ulothrix & 7 \\
\hline & Closterium & 47 \\
\hline & Cosmarium & 127 \\
\hline & Euastrum & 33 \\
\hline & Staurastrum & 100 \\
\hline & Staurodesmus & 29 \\
\hline \multirow{2}{*}{ Charophyceae } & Chara & 3 \\
\hline & Nitella & 2 \\
\hline
\end{tabular}


Algas dulceacuícolas de Chile: PARrA, O.

\section{BIBLIOGRAFIA}

Asprey, J.F., K. Benson-Evans \& J.E. Furet. 1964. A contribution to the study of South American Freshwater Phytoplankton. Gayana Bot. 10: 118.

Basualto, S., A. Acuña \& O. Parra. 1992. Contribución de biomasa fitoplanctónica del Río Bío-Bío en el Golfo de Arauco, VIII Región, Chile. Rev. Biol. Mar., Valparaíso, 27(2): 235-248.

Bicudo, C. E. \& D. Bicudo. 1996. Towards assaying biodiversity in freshwater algae. In: C. Bicudo $\&$ N. Menezes (eds.), Biodiversity in Brazil: a first approach. Sao Paulo CNPq: 5-16.

Borge, O. 1901. Sûsswasseralgen aus Sûdpatagonien. Bih. Kongl. Svenska. Vetensk. Akad. Handl. 27(1): 1- 40.

Bourrelly, P.C. 1972. Les algues d'eau douce: initiation a la systematique, I: les algues vertes. Paris: editions N. Boubèe \& Cie. 572 p.

Campos, H., W. Steffens, O. Parra, P. Domínguez \& AgüEro G. 1987a. Estudios limnológicos en el Lago Caburgua (Chile). Gayana Bot. 44: 63-86.

Campos, H., W. Steffens, G. Agüero, O. Parra O. \& L. ZÚNIIGA. 1987b. Limnology of Lake Riñihue. Limnológica 18(2): 339-345.

Campos, H., W. Steffens, G. Agüero, O. Parra \& L. ZÚÑIGA. 1988. Limnological study of Lake Llanquihue (Chile). Morphometry, physics, chemistry, plankton and primary productivity. Arch. Hydrobiol. 81(1): 37-67.

Campos, H., W. Steffens, G. Agüero, O. Parra \& L. ZúÑIGA. 1989. Estudios Limnológicos en el lago Puyehue (Chile). Morfometría, factores físicos y químicos, plancton y productividad primaria. Medio Ambiente 10(2):36-53.

Campos, H., W. Steffens, G. Agüero, O. Parra \& L. ZúNigA. 1990. Limnology study of lake Todos los Santos (Chile). Morphometry, Physics, Chemistry, Plankton and Primary Productivity. Arch. f. Hidrobiol. 117(4): 453-484.

Campos, H., W. Steffens, G. Agüero, O. Parra \& L. ZúNIIGA. 1992a. Limnological studies of lake Rupanco (Chile). Morphometry, Physics, chemistry, plankton and primary productivity. Archiv. f. Hydrobiol./Suppl. 90 (1):85-113.

Campos, H., W. Steffens, G. Agüero, O. Parra \& L. ZÚÑIGA. 1992b. Limnology of Lake Ranco (Chile). Limnológica 22(4): 337-353.

Campos, H., D. Soto, W. Steffens, O. Parra \& G. Agüero. 1994a. Limnological studies of lake Toro (Chile) from Patagonian of South America. Archiv. f. Hydrobiol. Suppl. 99(1/2): 199-215.

Campos, H., D. Soto., W. Steffens, O. Parra, G. Agüero \& L. ZÚÑIGA. 1994b. Limnological studies of Lake Sarmiento (Chile); A Subsaline lakes from Chilean Patagonian. Archiv. f. Hydrobiol. Suppl.99 (1/2):217-234.

Campos, H., D. Soto., O. Parra, W. Steffens \& G. Agüero. 1996. Limnological studies of Amarga Lagoon,
Chile: a saline lake in Patagonian of South America. Int. J. of Salt Lake Research. 4:301-14.

Cifuentes, A.S., M. González, M. Conejeros, V. Dellarossa \& O. Parra. 1992. Growth and carotenogenesis in eight strains of Dunaliella salina Teod. from Chile. Journ. of Applied Phycology 4: 111-118.

DADAY, E.V. 1902. Beitràge zur Kenntniss der SûsswasserMikrofauna von Chile. Termèszetr. Fûz. 25.

De Toni, G.B. 1889. Ûber einige Algen aus Feurland und Patagonien. Hedwigia 1889: 24-26.

Dellarossa, V., E. Ugarte \& O. Parra. 1976. Estudio limnológico de las lagunas "Chica de San Pedro", "La Posada" y "Lo Méndez" II. Aspectos cuantitativos del plancton invernal y su relación con algunas características físicas y químicas del ambiente. Bol. Soc. Biol. Concepción 50: 87-101.

Dorador C., R. Pardo \& I. Vila. 2003. Variaciones temporales de parámetros físicos, químicos y biológicos de un lago de altura: el caso del Lago Chungará. Revista Chilena de Historia Natural 76: $15-22$.

Espinosa, M. 1917. Los alerzales de Piuché. Bol. Mus. Nac. de Chile. 10: 82-83.

EspinOsA, M.R. 1923. Lista sistemática de algunas algas chilenas de agua dulce. Rev. Chilena Hist. Nat. 27: 93-95.

Forest, H. \& R. Weston. 1966. Blue green Algae from desert of Northern Chile. J. Phycol. 2: 163-164.

GaIN, L. 1911a. La neige verte et la neige rouge des regions antarctiques. Bull. Mus. Hist. Nat., Paris 17: 479482.

GAIN, L. 1911b. Note sur la flore algologique d'eau douce de l'Antarctique sud-americaine. Bull. Mus. Hist. Nat., Paris 17: 371-376.

Hammond, P.M. 1992. Systematics and diversity: species inventory. In: Groombridge, B. (ed.). Global diversity: status of the Earth's living resources. Report compiled by the World Conservation Monitoring Center. London: Chapman \& Hall: 17-39.

Hammond, P.M. 1995. Described and estimated species numbers: an objective assessment of current knowledge. In: Allsop, D., Colwell, R.R. \& Hawksworth, D.I. (eds.). Microbial diversity and ecosystem function. Cambridge: University Press: 29-71.

IzQuierdo, V. 1906. Ensayo sobre los Protozoos de las aguas dulces de Chile. Imprenta Cervantes, Santiago de Chile, 228 pp.

Mühlhauser, H., N. Hrepic, P. Mladinic, V. Montecino \& S. CABRERA. 1995. Water quality and limnological features of a high altitude Andean lake, Chungará in northern Chile. Revista Chilena de Historia Natural 68: 341-349.

Navarro, N. \& S. Avaria. 1971. Fitoplancton del lago Peñuelas. Anales Mus. Hist. Nat. Valparaíso 4: 287-338. 
Gayana 70(1), 2006

PARRA, O. 1973. Estudio cualitativo del fitoplancton de la Laguna Verde, Concepción, Chile, excl. diatomeas. Gayana Bot. 23:1-93.

Parra, O. 1975a. Desmidiáceas de Chile I. Desmidiáceas de la Región de Concepción y alrededores. Gayana Bot. 30. 1-91.

Parra, O. 1975b. Un nuevo e interesante género de Xanthophyta para Chile: Pseudostaurastrum Chodat. Bol. Soc. Biol. Concepción 49: 149- 151.

PArra, O. 1977a. Stylococcus aureus Chodat (Chromophyta-Chrysophyceae) epibionte de microalgas planctónicas. Bol. Soc. Biol. Concepción 51: 203-212.

PARRA, O. 1977b. Sobre la presencia de Tetrachloris merismopedioides Skuja (Cyanochloridaceae/ Chlorobacteriaceae). Bol. Soc. Biol. Concepción 51: 213-217.

Parra, O. 1977c. Desmidiáceas de Chile II. Nuevas desmidiáceas para la región de Concepción. Bol. Soc. Biol. Concepción 51: 193-201.

PARRA, O. 1989. La eutroficación de la Laguna Grande de San Pedro: Un caso de estudio. Ambiente y Desarrollo 5(1): 117-136.

PARRA, O. 1998. Una aproximación sistémica para la evaluación de la biodiversidad algal en ambientes acuáticos continentales de Chile. Sociedad Ficológica de América Latina y el Caribe, Sociedad Brasileña de Ficología. :167-178.

Parra, O. \& C. Bicudo. 1986. Groenbladia (Desmidiaceae): a first record of occurrence in Chile. Gayana Bot. 42 (3/4): 47-49.

Parra, O. \& C. M. Bicudo. 1996. Algas de Aguas Continentales: Introducción a la Biología y Sistemática. Ediciones Universidad de Concepción. 268 pp.

PARRA O. \& M. GonzÁlez. 1975. Synechocystis Savageau, Nuevo género de Cyanophyta para Chile. Bol. Soc. Biol. Concepción 49: 153-155.

PARRA, O. \& M. GonzÁLez. 1976. Guía bibliográfica y distribución de las cianófitas de Chile (excluyendo el Continente Antártico). Gayana Bot. 32: 1-55.

Parra, O. \& M. GonzÁlez. 1977a. Catálogo de las algas dulceacuícolas de Chile: Pyrrophyta, Chrysophyta Chrysophyceae, Chrysophyta-Xantophyceae, Rhodophyta, Euglenophyta y Chlorophyta. Gayana Bot. 33: 1- 102

Parra, O. \& M. GonzÁlez. 1977b. Desmidiáceas de Chile III. Desmidiáceas de la Isla de 34: 1- 103.

Parra, O. \& M. González. 1978. Freshwater algae of Chiloé Island. Nova Hedwigia 30: 873-924.

Parra, O. \& M. GonzÁlez. 1982. Freshwater algae of Juan Fernández Island (Chile) I. Nova- Hedwigia 38 (2/4): 1-20.

Parra, O., C. Jara \& L. GuZmán. 1989. Las lagunas intraurbanas de Concepción: Estado actual y perspectivas de recuperación y uso. Actas del III Encuentro Nacional del Medio Ambiente.: 301-313.

Parra, O. \& V. Dellarossa. 1987. Perfil biológico de un bloom de una microalga en Laguna Redonda, Concepción, Chile. Bol. Inf. Limnológico 9:4-19.
Parra, O., D. Avilés, J. Becerra, V. Dellarossa \& R. MonToya. 1986. First toxic blue-green algal bloom recorded for Chile: a preliminary report. Gayana Bot. 43 (1-4): 15-17.

Parra, O., C. Valdovinos, R. Urrutia, M. Cisternas, E. HaBIT \& M. MARDONES. 2003. Caracterización y tendencias tróficas de cinco lagos costeros de Chile central. Limnetica 22 (1-2): 51-83.

Parra, O., E. Ugarte, L. Balabanoff, S. Mora, M. Liebermann \& A. Aron. 1980. Remarks on a bloom of Microcystis aeruginosa Kuetzing. Nova Hedwigia 33: 971-1004.

Parra, O., H. González \& M. GonzÁlez. 1984. A comparison of epiphytic diatom assemblages attached to filamentuos algae in lotic freshwater habitats of Chile. Gayana Bot. 41 (1/2): 85-117.

Parra, O., M. González, V. Dellarossa, A.S. Cifuentes \& M. Conejeros. 1990. Caracterización biológica de una cepa chilena de Dunaliella salina potencialmente comerciable. Arch. Biol. y Med. Exp. 23:141-146.

Parra, O., M. González, V. Dellarossa, P. Rivera \& M. Orellana. 1982-1983. Manual Taxonómico del Fitoplancton de Aguas Continentales; con especial referencia al fitoplancton de Chile. Editorial de la Universidad de Concepción Vol. 1, Cyanophyceae, 1982; Vol. 2, ChrysophyceaeXanthophyceae, 1982; Vol. 3, Cryptophyceae, Dinophyceae y Euglenophyceae, 1982 ; Vol. 4, Bacillariophyceae, 1982; Vol. 5 (partes 1 y 2), Chlorophyceae, 1983.

Parra, O., S. Basualto, R. Urrutia \& C. Valdovinos. 1999. Estudio comparativo de la diversidad fitoplanctónica de cinco lagos de diferentes niveles tróficos. Gayana 56(2): 25-40.

Parra, O., S. Basualto, R. Urrutia, D. Avilés \& P. Rivera. 1993. Composición taxonómica del fitoplancton y fitobentos del río Biobío, Concepción, Chile. En: Evaluación de la Calidad del Agua y Ecología del Sistema Limnético y Fluvial del río Bío-Bío. (Eds. F. Faranda y O. Parra), Serie Monografías Científicas EULA 12: 189-216.

Parra, O., V. Dellarossa \& E. Ugarte. 1976. Estudio limnológico de las lagunas Chica de San Pedro, La Posada y Lo Méndez I. Análisis cuali y cuantitativo del plancton invernal. Bol. Soc. Biol. Concepción 50: 73-86.

Pereira, I. \& O. Parra. 1984. Algas filamentosas dulceacuícolas de Chile I. Algas bentónicas de la Región de Concepción. Gayana Bot. 41 (3/4): 201-224.

Pérez Canto, J. 1929. Los protozoarios del suelo. Rev. Chilena Hist. Nat., 33: 146-148.

Rivera P., O. Parra \& M. GonzÁlez. 1973. Fitoplancton del Estero Lenga Chile. Gayana Bot. 23: 1-93.

Round, F.E. 1973. The biology of algae. London: Edward Arnold Publications. 260 pp.

SchwaBe, G.H. 1936. Sobre biotopos termales en el Sur de Chile. Bol. Soc. Biol. Concepción 10(2): 93-123. 
Soto, D., H. Campos, O. Parra, L. ZúÑIga \& W. StefFens. 1994. The Torres del Paine Lake District (Chilean Patagonian): A case of pristine N-limited lakes and ponds. Archiv. f. Hydrobiol. Suppl. 99 (1/ 2): 181-197.

Theoduloz, C. \& O. Parra. 1984. Desmidáceas de Chile IV. Desmidiáceas del sistema de lagos de la Cordillera del Paine y alrededores. Gayana Bot. 41 (3- 4): 141-200.

Thomasson, K. 1955. Studies on South American Freshwater Plankton from Tierra del Fuego and Valdivia. Acta Horti Gotonb. 19: 193-225.

Thomasson, K. 1963. Araucanian lakes. Plankton studies in North Patagonien with notes on terrestrial vegetation. Acta Phytogeogr. Suec. 47: 1-139.

Vila, I., I. BARENDS \& V. MonTeCinO. 1987a. Composición, abundancia y distribución espacial y temporal del fitoplancton del embalse Rapel (1976-1977). Revista Chilena Historia Natural 60: 37-55.

Vila, I. \& H. Mühlhauser. 1987b. Dinámica de lagos de altura, perspectivas de investigación. Archivos de Biología y Medicina Experimentales (Chile) 20: 95-103.

Vila, I., M. Contreras \& J. Pizarro. 1997. Eutrophication and phytoplankton selective responses in a temperate reservoir. Verh. Internat. Verein. Limnol. 26: 798-802.

VILA, I. \& R. PARDo. 2003. Respuesta de la estructura fitoplanctónica a las perturbaciones antrópicas de un lago templado. Limnetica 22(1-2): 93-102.

Villalobos, L., O. Parra, M. Grandjean, E. Jaque, S. Woelfl. \& H. CAMPos. 2003. A study of the river basins and limnology of five humic lakes on Chiloé Island. Revista Chilena de Historia Natural 76: 563-590.

Villalobos, L., S. Woelfl, O. Parra, W. Steffen, G. Agüero \& H. Campos. 2003. Lake Chapo - a baseline study on a deep, oligotrophic northpatagonian lake prior to its use for hydroelectricity generation: II. Biological properties. Hydrobiologia 510: 225-237.

WiLle, N. 1884. Bidrag till Sydamerikas Aldflora 1-3. Bih. Kongl. Svenska Vetensk. Akad. Handl. 8(18): 1-64.

Wilson, E.O. 1992. The diversity of life. Cambridge, massachusetts: Belknap Press. 223 pp.

Woelfl, S. L. Villalobos, O. Parra, W. Steffen \& H. CAmpos. 2003a. Lake Chapo- a baseline study on a deep, ultraoligotrophic, northpatagonian lake prior to hydroelectrical use: I. Physical and chemical. Hydrobiologia 510: 217-224.

Woelfl, S., L. Villalobos \& O. Parra. 2003b. Trophic parameters and method validation in Lake Riñihue (North Patagonia, Chile) from 19781997. Revista Chilena de Historia Natural 76(3): 459-474. 